\title{
The Oxidative Stress Markers of Horses-the Comparison with Other Animals and the Influence of Exercise and Disease
}

\author{
Saori Shono ${ }^{1}$, Azusa Gin ${ }^{1}$, Fumiko Minowa ${ }^{2}$, Kimihiro Okubo ${ }^{3}$ and Mariko Mochizuki ${ }^{1, *}$ \\ 1 Department of Applied Science, School of Veterinary Nursing and Technology, Nippon Veterinary and Life \\ Science University, Tokyo 180-8602, Japan; sshono@nvlu.ac.jp (S.S.); ginnnn525@yahoo.co.jp (A.G.) \\ 2 Minowa Horse Clinic, Tokyo 144-0033, Japan; hgf-mino@y9.dion.ne.jp \\ 3 Department of Otolaryngology, Graduate School of Medicine, Nippon Medical School, Tokyo 113-8602, \\ Japan; ent-kimi@nms.ac.jp \\ * Correspondence: m-mochi@nvlu.ac.jp; Tel.: +81-422-31-4151
}

Received: 28 February 2020; Accepted: 2 April 2020; Published: 3 April 2020

check for updates

Simple Summary: Although oxidative stress is detrimental in biological systems, direct analysis of the active oxygen species that causes the stress has been difficult at a clinical level. In the current study, we analyzed the levels of diacron-reactive oxygen metabolites (d-ROMs) and biological antioxidant potential (BAP) in the serum of horses. These are easy to measure and they provide information on the level of oxidative stress in an animal. The mean d-ROM level in horses was higher than those in dogs or dairy cattle, and the levels in horses can be used to distinguish those with a disease.

\begin{abstract}
Diacron-reactive oxygen metabolite (d-ROM) and biological antioxidant potential (BAP) levels in the serum of horses were measured (ponies, $n=15$; thoroughbred, $n=31$; other full-sized horses, $n=7)$. The mean d-ROM levels in horses were significantly higher $(p<0.001)$ than those in dairy cattle $(n=25)$ and dogs $(n=31)$. However, d-ROM levels in horses were lower than the standard levels reported in humans. When d-ROM and BAP levels were plotted graphically, the points for horses with a disease (ringbone in 1 Japanese sports horse, cellulitis in 1 thoroughbred, melanoma in 1 Lipizzaner) fell outside the group of points for other (non-diseased) horses. A similar separation was seen (using data from other authors) for a horse with Rhodococcus equi, a horse following castration surgery, and a mare following delivery. These results, comparing horses, other animals, and humans, are interesting from the standpoint of comparative medicine, and they contribute to the sparse literature available on d-ROM and BAP levels in animals. Because the level of d-ROM and BAP levels were changed depending on the situation of health, those indexes are promising as indices of health in horses.
\end{abstract}

Keywords: diacron-reactive oxygen metabolite; biological antioxidant potential; horse; oxidative stress index

\section{Introduction}

Oxidative stress is detrimental to biological systems. It occurs when the balance between reactive oxygen species and antioxidants is disturbed. In humans, it is well known that oxidative stress is associated with various diseases, including diabetes [1], obesity [2], inflammation, and cancer [3], and with lifestyle habits such as fitness activities [4], alcohol consumption [5] and smoking [6]. There have also been many studies in various animals using indexes related to oxidative stress and antioxidant activity such as 8-OHdG [7] and lipid peroxidation [8]. The aims of those studies were to cover a lot 
of ground, for example, animal welfare [9,10], produce of value-added animal product [11], efficient produce of animal product [7], and estimation of disease [12].

Because of their high activity and short half-life, direct analysis of the reactive oxygen species that cause oxidative stress has been difficult at the clinical level; but more recently, the equipment has improved, and the measurement of oxidative stress levels is relatively easy. The recent method involves measuring hydroperoxides $(\mathrm{ROOH})$ levels as metabolic products to determine the level of oxidative stress as diacron-reactive oxygen metabolites (d-ROMs). Because it is easy to maintain and operate, the measuring equipment has been used in relation to human diseases in clinical practice, with reports appearing on cardiovascular disease [13], high-density lipoprotein [14], and pediatric oncology [15], although there are few published studies using this instrument on animals.

In the study related animals, the d-ROMs concentration has been used as an index of stress. Fazio et al. [9] reported that higher d-ROMs concentration was observed in dogs due to road transportation. In the study using dogs protected in a shelter, d-ROMs concentration of serological negative dogs was significantly higher than that of serological positive dogs [10]. Oxidative stress is also monitored for health management in athletes [16]. As with athletes, racehorses and competition horses experience high levels of physical exercise, and although knowledge of the status of d-ROMs levels in the serum of horses might provide important information on their condition, there is little research published on the same. Further, not only for horses, there is also little information about the data of other animals such as dogs. To know the character of data of d-ROMs in the serum of horses, the comparison with the results of other animals is thought to be interesting. Thus, in the present study, the concentration of d-ROMs in the serum of horses is compared with that in other animals (dairy cattle and dogs) for the influence of exercise and disease on d-ROM levels. Then, the influence on d-ROMs of exercise load and diseases in horses is investigated. Finally, we investigate antioxidant capacity by measuring biological antioxidant potential (BAP).

\section{Materials and Methods}

\subsection{Animals and Sample Preparation}

The study was approved by the ethical policies of experimental animals of Nippon Veterinary and Life Science University (approval number, daily cattle: 28K-20, S28K-20; horse: 27S-8, 28K-19 and 29; dogs: 28S-9, S28-9). Serum samples were obtained from horses, dairy cattle, and dogs. The serum samples of horses were collected at an equestrian club in Shizuoka Prefecture in Japan on 19 August 2015 during separate research (ponies; $n=12$, thoroughbreds; $n=26$ ) (Red symbols in Figures 1-3). Three ponies and four thoroughbreds in this club with decreased physical activity (mean value of these 7 animals was indicated in [17]) are shown by black symbols in Figure 2. The information on the horses (such as sex and age) were reported previously [17]. A total of 8 full-sized horses fed in the same club are newly added in this study, as follows: thoroughbred (1 gelding, age $20 \mathrm{y})$, Selle Français (2 females, average age 12 y; 2 geldings, average age 13.5 y), KWPN (1 gelding, age 15 y), Anglo-Arabian (1 gelding, age 22 y), DWB (1 gelding, 23 y). Those 8 full-sized horses are manifested in red symbols in Figures 1-3 (thoroughbred, circle; others, triangle). Those samples were also collected on 19 August 2015. Horses at an equestrian club in Shizuoka Prefecture that were suffering from disease were also sampled: Japan sports horse species (gelding, $10 \mathrm{y}$, ringbone), thoroughbred (gelding, 26 y, cellulitis), and a Lipizzaner (gelding, 30 y, melanoma) (see black symbols in Figure 3). On the day of sampling, the horses were fed at 6:00 a.m. and then pastured between 7:30 and 10:00 a.m. Samples were collected between 10:30 a.m. and 12:00 noon before the horses were used for horseback riding. Serum was collected from a total of 27 dairy cattle consisting of Holstein Friesian $(n=25$, average age $52.72 \pm 3.20 \mathrm{~m}$; green circle symbols in Figure 1$)$, Jersey $(n=2$, average age $59.97 \mathrm{~m}$; green square symbol in Figure 1) and Brown Swiss ( $n=1$, age $62.2 \mathrm{~m}$; green triangle symbols in Figure 1$)$ at a farm in Yamanashi Prefecture attached to the Nippon Veterinary and Life Science University. The samples from the dairy cattle were collected during another investigation related to animal-assisted education 
on a farm [18]. In the current day of sampling on 9 May 2016, cattle were fed at 5:00 a.m. The milking was terminated at 6:30 a.m. Sampling was conducted between 10:00 and 11:00 a.m. Blood samples (20 $\mathrm{mL}$ ) from the horses and dairy cattle were collected into vacuum blood collection tubes (Venoject II, Terumo Corporation, Tokyo, Japan). After coagulation had taken place, the samples were centrifuged at $3000 \mathrm{rpm}(1468 \times g)$ for $15 \mathrm{~min}$. The supernatants were collected into sample tubes (BM Equipment Co., Ltd., Tokyo, Japan). Except for the horses having disease used in Figure 3, all animals, including cattle, dogs, and horses, were not in treatment due to disease.

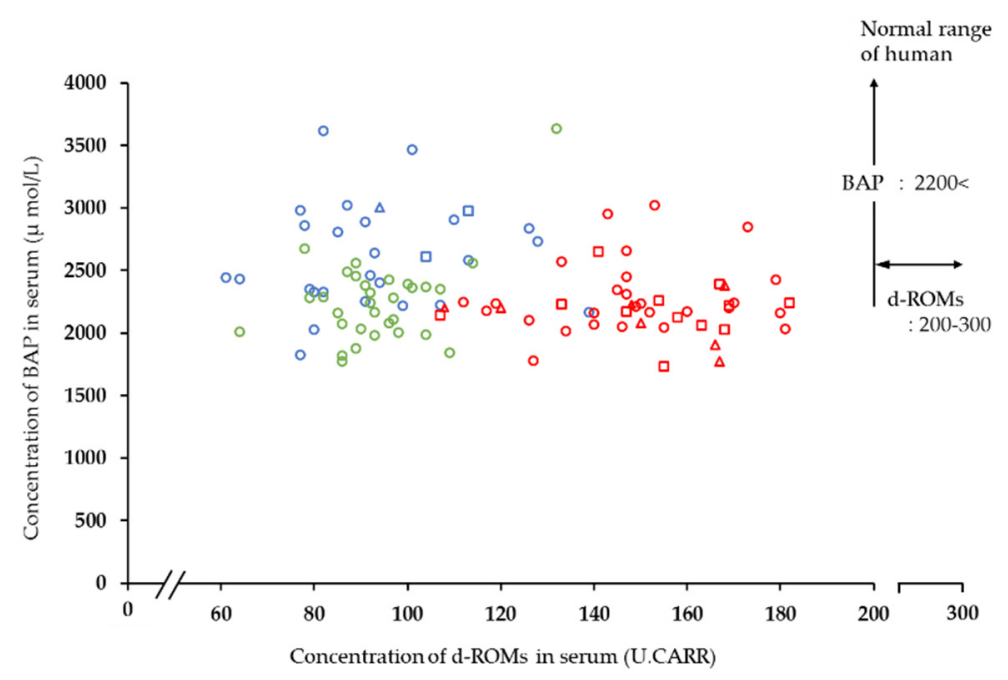

Figure 1. Comparison between diacron-reactive oxygen metabolite ( $\mathrm{d}-\mathrm{ROM})$ and biological antioxidant potential (BAP) concentrations in serum of horses in an equestrian club in Shizuoka Prefecture and that of other animals (dairy cattle and dogs). Red symbols: horses in an equestrian club in Shizuoka Prefecture used in our study (circle: thoroughbred; square: pony; triangle: full-sized horses other than thoroughbred). Blue symbols: dairy cattle (circle: Holstein Friesian; triangle: Brown Swiss; square: Jersey). Green symbols: dogs.

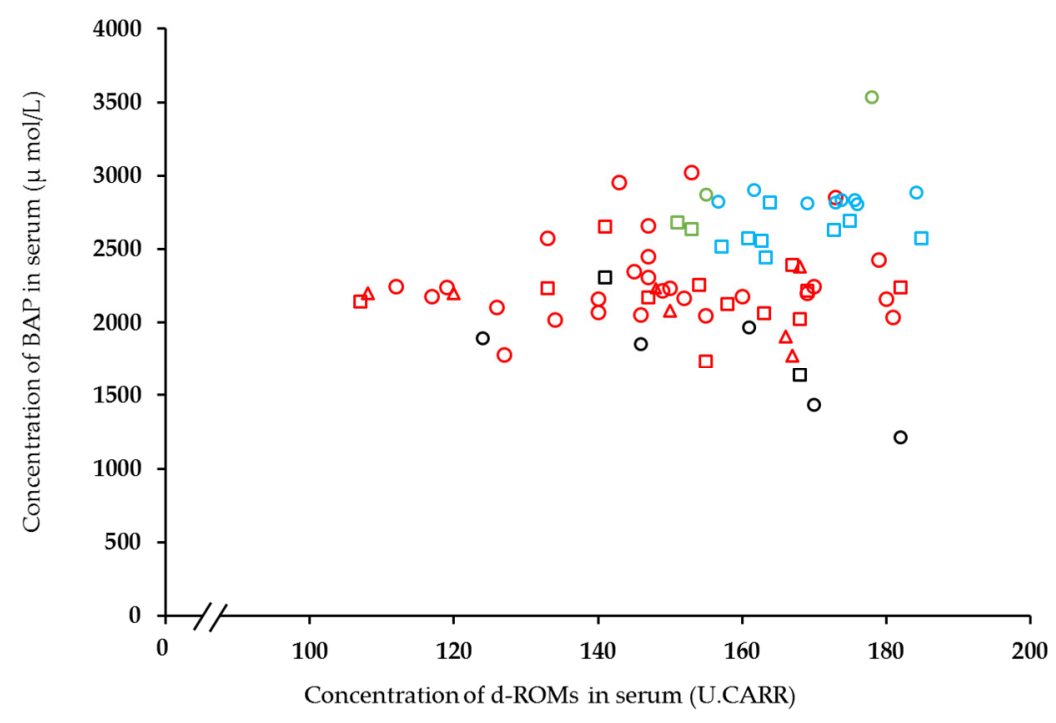

Figure 2. Comparison between d-ROM and BAP concentrations in serum of horses in an equestrian club in Shizuoka Prefecture and that of race and endurance horses. Red and black symbols: horses in an equestrian club in Shizuoka Prefecture in our study (circle: thoroughbreds; square: ponies; triangle: full-sized horses other than thoroughbreds). Black symbols indicate horses with decreased physical activity (mean value of these 7 animals was indicated in [17]). Blue symbols: endurance horses [19] (square: before a race; circle: after a race). Green symbols: racehorses (thoroughbreds) (square: before simulated race; circle: after simulated race) [20]. 




Figure 3. Comparison between d-ROM and BAP concentrations in serum of horses in an equestrian club in Shizuoka Prefecture and that of horses suffering disease or other stress. Red symbols: horses in an equestrian club in Shizuoka Prefecture in our study (circle: thoroughbreds; square: ponies; triangle: full-sized horses other than thoroughbreds). Black symbols: horses suffering disease or other stress (square: horses in our study; other symbols: data from previous reports [21-23]).

Frozen serum or plasma of dogs after examination at an animal hospital in Tokyo were transported immediately to the laboratory of Nippon Veterinary and Life Science University. Those samples of dogs were residue samples of the health exam in an animal hospital. Although the time of blood sampling varied, the animal hospital has recommended to at least eat nothing $8 \mathrm{~h}$ before the health exam. The sample of dogs was collected between April and July 2017. The time and day of sampling were not consolidated due to a health exam. A total of 31 samples were obtained from toy poodles. Information about these dogs is summarized in Table 1. The serum samples in tubes were stored at $-30{ }^{\circ} \mathrm{C}$ in a freezer (Panasonic Healthcare Co. Ltd., Tokyo, Japan) until analysis.

Table 1. Information on the dogs used in the present study.

\begin{tabular}{ccccc}
\hline Breed & Sex & $\boldsymbol{n}$ & Age (Months) & Weight (Kg) \\
\hline \multirow{3}{*}{ Toy poodle } & Female & 6 & $47.17 \pm 26.52$ & $4.43 \pm 0.80$ \\
& Neuter & 14 & $47.14 \pm 11.37$ & $5.38 \pm 0.46$ \\
& Spay & 11 & $46.18 \pm 13.37$ & $3.85 \pm 0.42$ \\
\cline { 2 - 5 } & & 31 & $46.81 \pm 8.31$ & $4.65 \pm 0.29$ \\
\hline
\end{tabular}

Data are presented as mean \pm standard error of the mean. $n$ : number of samples.

\subsection{Data on Horses from Previous Studies}

Some of the data on the horses in this study were taken from previous studies: the data obtained from endurance horses before (green square symbols in Figure 2) and after (green circle in symbols Figure 2) an endurance race [19], and data for thoroughbreds before and after exercise using a treadmill [20] were used as a model for healthy horses that experienced increased exercise.

Data from some special cases were also included: horses with Rhodococcus equi [21], after delivery [22], and after castration surgery [23] (black symbols in Figure 3). 


\subsection{Measurement}

Because the retention period of stored samples is recommended until 1 year under less than $-20{ }^{\circ} \mathrm{C}$ in the manual provided by the manufacturer, the stored samples were analyzed within a time limit of the storage period. On the day of analysis, samples were transferred to an ice chamber to thaw.

The concentration of d-ROMs and BAP in serum was analyzed using a free radical analyzer (Free Carpe Diem, Diacron International srl., Grosseto, Italy). The oxidative stress index (OSI) was calculated using the formula $(\mathrm{d}-\mathrm{ROM} / \mathrm{BAP} \times 100)$.

\subsection{Statistical Methods}

Data are presented as mean \pm standard error of the mean. Analyses were performed using the Japanese language version of Lotus 2001 software (Microsoft Japan Co., Ltd., Tokyo, Japan) and Excel 2016 (Microsoft Japan Co., Ltd., Tokyo, Japan). The normality of data has been checked by the Kolmogorov-Smirnov test and the Shapiro-Wilk test. Because the data in the present study has shown a mixture of non-normal and normal and distribution, significant differences in elemental concentrations were tested for using the Kruskal-Wallis test. In addition, the Dunn test was used as a post hoc test. The differences in 2 variates were tested for using the Mann-Whitney $U$-test. Those statistical tests were performed using the Japanese language version of IBM statistics 19 (IBM Japan Ltd., Tokyo, Japan) and Bell curve (Social Survey Research Information Co., Ltd., Tokyo, Japan).

\section{Results}

\subsection{Comparison between Horses and Other Animals}

The concentration of d-ROMs and BAP in the serum of horses and other animals are shown in Table 2 and Figure 1. The value for horses was significantly higher than for both dairy cattle $(p<0.001)$ and dogs $(p<0.001)$. However, horse $\mathrm{d}-\mathrm{ROM}$ values in the present study were lower than the reference value for $\mathrm{d}-\mathrm{ROM}$ in humans.

Table 2. d-ROM and BAP levels in animal sera and oxidative stress index (OSI).

\begin{tabular}{|c|c|c|c|c|c|}
\hline Animal & $n$ & $\begin{array}{l}\text { d-ROMs } \\
\text { (U.CARR) }\end{array}$ & $\begin{array}{c}\text { BAP } \\
(\mu \mathrm{mol} / \mathrm{L})\end{array}$ & OSI & Figure \\
\hline Dog & 31 & $93.74 \pm 2.20^{b}$ & $2258.38 \pm 62.34^{b}$ & $4.20 \pm 0.11^{b}$ & Green symbols in Figure 1 \\
\hline Horse & 46 & $149.24 \pm 2.92^{\mathrm{a}}$ & $2237.70 \pm 39.71^{b}$ & $6.76 \pm 0.18^{a}$ & Red symbols in Figure 1 \\
\hline Dairy cattle & 28 & $93.48 \pm 3.42^{b}$ & $2622.09 \pm 78.09^{a}$ & $3.65 \pm 0.17^{b}$ & Blue symbols in Figure 1 \\
\hline
\end{tabular}

The mean serum BAP concentrations in the horses, dairy cattle and dogs were 2237.70, 2622.09 [18], and $2227.81 \mu \mathrm{mol} / \mathrm{L}$, respectively. The value for dairy cattle was significantly higher than for both horses $(p<0.001)$ and dogs $(p<0.001)$, but there was no significant difference $(p=0.96)$ between values for dogs and horses. Due to the higher d-ROM value, the OSI for horses was significantly higher $(p<0.001)$ than for both dogs and dairy cattle.

\subsection{Comparisons Among Horses}

The concentrations of $\mathrm{d}-\mathrm{ROM}$ and BAP in the serum of riding horses in an equestrian club and race and endurance horses are shown in Figure 2. There were seven animals with decreased physical activity due to old age or exercise limitation (black symbols in Figure 2) for which the BAP level (mean: $1758.30 \mu \mathrm{mol} / \mathrm{L}$ [17]) was lower than that of normal horses. In contrast, d-ROM and BAP levels tended to be higher in racehorses (green symbols in Figure 2) and endurance horses (blue symbols in Figure 2). 
When horses were considered in 3 categories, horses in equestrian club $(n=46)$, before endurance race $(n=10)$ and simulated race $(n=2)$, after endurance race $(n=10)$ and simulated race $(n=2)$, a significant difference $(p<0.01)$ were obtained in d-ROMs concentrations in the serum of horses in an equestrian club than of other two groups. A similar significant difference $(p<0.001)$ was also obtained in the investigation of BAP. The significant difference $(p<0.001)$ between before race and after race was obtained on only the BAP level.

Values for d-ROMs and BAP in the serum of horses suffering from a disease, such as ringbone, melanoma, Rhodococcus equi [21], or cellulitis, were distributed away from the values for normal horses, as were the values for a horse after delivery [22] and a horse after castration surgery [23] (Figure 3). There is a significant difference $(p<0001)$ between the mean concentration of d-ROMs in the serum of horses having disease $(n=4)$ and that of horses having no disease $(n=46)$. A similar tendency $(p<0.05)$ was also obtained on the investigation of BAP.

\section{Discussion}

In the first experiment in the present study, we compared d-ROM and BAP concentrations in serum of horses versus dogs and dairy cattle. In the study using healthy Labrador dogs, it was reported that the concentration of $\mathrm{d}-\mathrm{ROMs}$ and BAP were $73.9 \pm 8.78 \mathrm{UCARR}$ and $2446 \pm 585 \mu \mathrm{mol} / \mathrm{L}$, respectively [24]. The results of d-ROMs in the study [24] were lower than those in our study. We also analyzed d-ROMs and BAP concentration in serum of Labrador dogs $(n=4)$, the data (d-ROMs; 70.25 UCARR, BAP; $2121.35 \mu \mathrm{mol} / \mathrm{L}$ ) were similar with the study by Pasquini et al. [24]. It was thought that there are differences depending on the breed. In the previous study related to the dairy cattle $(n=74)$, it was reported that $\mathrm{d}-\mathrm{ROMs}$ and BAP concentration was $158 \pm 23.0 \mathrm{UCARR}$ and $2558 \pm 108.5 \mu \mathrm{mol} / \mathrm{L}$, respectively. The result of d-ROMs was slightly lower than that of the previous study [25].

The d-ROM concentrations in horses were significantly higher $(p<0.001)$ than in the other animals. In reports on human health, d-ROM levels are increased due to lifestyle habits such as fitness activities [4], alcohol consumption [5], and smoking [6]. Clearly, because animals are not in the habit of smoking or drinking, the higher d-ROM levels in horses must be influenced by other factors. Because the amount of exercise performed by dairy cattle and dogs was thought to be obviously less than that of horses, the results suggest that the exercise amount is one reason for the higher d-ROM levels in horses. The density of oxygen-carrying erythrocytes in the blood of horses is known to be greater than in other animals such as dogs and cattle [26]. Further, the concentration of hemoglobin in the blood is increased depending on exercise intensity due to an increase in the blood cell:volume ratio. Thus, it is possible that horses maintain their blood oxygen at the higher level required for exercise [26]. Because the level of $\mathrm{d}$-ROMs after a race in the blood of athletes in various sports such as a race of duathlon [27], mountain biking [16], who consume more oxygen, is invariably higher than before the race, the basis of the higher $\mathrm{d}-\mathrm{ROM}$ concentration in serum of horses may be for the same reason.

Then, we compared d-ROM concentration in the serum of horses owned by an equestrian club against race and endurance horses. The d-ROM levels of the race and endurance horses were relatively high and focused, while those of the horses in the equestrian club were more widely distributed. The horses from the equestrian club varied in age and breed, and the amount of exercise they received differed depending on age and role, and it is probably for these reasons that their d-ROM levels fell over a wider range. The BAP concentration in serum of race and endurance horses was higher than in the other horses in this study. Because a higher BAP level was also reported in the other study using thoroughbred racehorses [28], it is suggested that the differences in exercise significantly influence the level of OS biomarkers. It has been reported [29] that the plasma BAP concentration in active older people is significantly higher than in the inactive elderly. Further, in a study of triathletes of the duathlon (running, cycling, running), d-ROM levels after the first run were significantly higher than levels observed before the race. However, because BAP levels also increased, the overall effect was that OSI gently decreased during the race [28]. The mean OSI in horses in the present study (range: 5.54-7.19) was similar to that of endurance horses [19], although considerable individual variability 
(range: 4.84-9.42) was observed in the horses in the present study. It has been suggested [30] that there is a significant negative correlation between age and BAP concentration in the serum of older adults. Further, a significant correlation was also observed between the ratio between d-ROMs and BAP. Thus, it is possible that BAP and OSI levels decrease with age. In the study using thoroughbred racehorses [28], it was also suggested that the higher BAP level and lower OSI are reported in low age of female horses. However, the decreasing trend of BAP level in male horses is not marked than that of females in this report. Because this study suggests that there is a gender difference in the level of BAP, an investigation depending on the gender difference is necessary in further study. Because the age of the horses at the equestrian club was obviously higher than that of the dairy cattle, the higher OSI, due to lower BAP levels in the serum of the horses, was thought to be age-related.

The concentrations of d-ROMs and BAP in the serum obtained from horses whose circumstances were other than normal were investigated in the present study. The horse diseases witnessed (ringbone, cellulitis, and melanoma) were chronic and serious illnesses, especially for the horse with melanoma, which was in a terminal condition. In humans, it has been suggested that changes in d-ROM levels are indicative of disease conditions [1-6]. Results from the present study also suggest that this index could be useful for the early detection of disease and/or disease conditions in horses. d-ROM levels for horses having no disease but having experienced castration surgery or delivery were also distributed outside the normal range, indicating that it is not only disease that is reflected in d-ROM and BAP levels in serum. Although the synovial fluid was used in the study, d-ROMs and OSI levels in the synovial fluid of the arthritis group were significantly higher than the control group [12]. Because the study of dairy cattle also found higher concentrations of d-ROMs and lower concentrations of BAP in dairy cattle having left displacement of abomasum, it was suggested, when compared to the control group [25], that several diseases are thought to influence d-ROMs concertation of animals. It has also been suggested that d-ROMs concentration rises under stress such as transportation [9] and shelter [10] in the studies using dogs. Because it is well known from long ago that there are horses feeling stress [31], the influence of stress should be also investigated on the horses. Because there are few reports to date on d-ROM and BAP levels in horses, further study would be necessary to describe more precisely how $\mathrm{d}-\mathrm{ROM}$ and BAP levels change under unusual conditions. Nevertheless, measurement of d-ROM and BAP levels are a useful indicator of health in horses.

\section{Conclusions}

The serum d-ROM and BAP levels in horses differ from that in dogs and dairy cattle. This study suggests that $\mathrm{d}-\mathrm{ROM}$ and BAP levels may be distributed over a wide range depending on the species. The data is interesting from the viewpoint of comparative medicine. Knowledge of d-ROMs and BAP in horses may help in studies on horses. Further detailed studies investigating more animal species would be desirable.

Author Contributions: Although K.O. is an expert at Otolaryngology, he is vice-president of the Japan Equestrian Federation and president of the Tokyo Equestrian Federation. Thus, he joined this study. Conceptualization, M.M., F.M., and K.O.; Methodology, M.M., and K.O.; Investigation, M.M., F.M., and A.G.; Formal analysis, M.M., A.G., and S.S.; Data curation; S.S., A.G., and M.M.; Writing-original draft in English, S.S. and M.M.; Writing-review and editing, K.O., F.M., A.G. and M.M.; Supervision, M.M; Funding acquisition, M.M. All authors have read and agreed to the published version of the manuscript.

Funding: Blood sample of horses was supported by a Grant-in Aid (No. 23580430) from the Ministry of Education, Science, Sports, Culture and Technology of Japan.

Acknowledgments: Samples were collected from animals by the following persons; Yasuhiro Nakajima, Kumi Sato, and Yuko Yokoi at the Palomino Pony Club in Shizuoka Prefecture in Japan; Mitsuho Kikuchi, Yukino Kikuchi, and Saki Nemoto at the Famille Animal Hospital in Tokyo, Japan; Takashi Teraoka, Seisuke Muramatsu, and Itaru Yoshimura at a farm in Yamanashi Prefecture attached Nippon Veterinary and Life Science University; Ryotaro Miura and Hisashi Mizutani at the Nippon Veterinary and Life Science University. The authors wish to thank these people for their help in sample collection. We also express thanks to Akihiro Saito at the Shizuoka Institute of Science Technology for sample preparation of the horses. 
Conflicts of Interest: The authors declare no conflicts of interest. The funders had no role in the design of the study; in the collection, analyses, or interpretation of data; in the writing of the manuscript, or in the decision to publish the results.

\section{References}

1. Asmat, U.; Abad, K.; Ismailb, K. Diabetes mellitus and oxidative stress-A concise review. Saudi Pharm. J. 2016, 24, 547-553. [CrossRef]

2. Furukawa, S.; Fujita, T.; Shimabukuro, M.; Iwaki, M.; Yamada, Y.; Nakajima, Y.; Nakayama, O.; Makishima, M.; Matsuda, M.; Shimomura, I. Increased oxidative stress in obesity and its impact on metabolic syndrome. $J$. Clin. Invest. 2004, 114, 1752-1761. [CrossRef]

3. Reuter, S.; Gupta, S.C.; Chaturvedi, M.M.; Aggarwal, B.B. Oxidative stress, inflammation, and cancer: How are they linked? Free Radic. Biol. Med. 2010, 49, 1603-1616. [CrossRef]

4. Finkler, M.; Lichtenberg, D.; Pinchuk, I. The relationship between oxidative stress and exercise. J. Basic Clin. Physiol. Pharmacol. 2014, 25, 1-11. [CrossRef]

5. Wu, D.; Cederbaum, A.I. Alcohol, Oxidative Stress, and Free Radical Damage. National institute on alcohol abuse and alcoholism, National Institutes of Health. U.S. In Department of Health and Human Services. Available online: https://pubs.niaaa.nih.gov/publications/arh27-4/277-284.htm (accessed on 1 December 2019).

6. Barreiro, E.; Peinado, V.; Galdiz, J.B.; Ferrer, E.; Marin-Corral, J.; Sánchez, F.; Gea, J.; Barberà, J.A. ENIGMA in COPD Project. Cigarette smoke-induced oxidative stress: A role in chronic obstructive pulmonary disease skeletal muscle dysfunction. Am. J. Respir. Crit. Care Med. 2010, 15, 477-488. [CrossRef]

7. Leskovec, J.; Rezar, V.; Svete, A.N.; Salobir, J.; Levart, A. Antioxidative effects of olive polyphenols compared to vitamin E in piglets fed a diet rich in N-3 PUFA. Animals 2019, 6, 161. [CrossRef]

8. Bekenev, V.; Garcia, A.; Hasnulin, V. Adaptation of piglets using different methods of stress prevention. Animals 2019, 9, 161. [CrossRef]

9. Fazio, F.; Casella, S.; Giannetto, C.; Giudice, E.; Piccione, G. Characterization of acute phase proteins and oxidative stress response to road transportation in the dog. Exp. Anim. 2015, 64, 19-24. [CrossRef]

10. Passantino, A.; Quartarone, V.; Pediliggeri, C.M.; Rizzo, M.; Piccione, G. Possible application of oxidative stress parameters for the evaluation of animal welfare in sheltered dogs subjected to different environmental and health conditions. J. Vet. Behav. 2014, 9, 290-294. [CrossRef]

11. Pokorska, J.; Kułaj, D.; Piestrzyńska-Kajtoch, A.; Radko, A. Impact of bovine lipocalin-2 gene on the antioxidant activity of milk from Polish Holstein-Friesian cows. Animals 2019, 9, 992. [CrossRef]

12. Tsuzuki, N.; Kanbayashi, Y.; Kusano, K. Markers for oxidative stress in the synovial fluid of Thoroughbred horses with carpal bone fracture. J. Equine Sci. 2019, 30, 13-16. [CrossRef] [PubMed]

13. Masaki, N.; Sato, A.; Horii, S.; Kimura, T.; Toya, T.; Yasuda, R.; Namba, T.; Yada, H.; Kawamura, A.; Adachi, T. Usefulness of the d-ROMs test for prediction of cardiovascular events. Int. J. Cardiol. 2016, 222, 226-232. [CrossRef] [PubMed]

14. Ito, F.; Ito, T.; Suzuki, C.; Yahata, T.; Ikeda, K.; Hamaoka, K. The application of a modified d-ROMs test for measurement of oxidative stress and oxidized high-density lipoprotein. Int. J. Mol. Sci. 2017, 18, 454. [CrossRef] [PubMed]

15. Nishikawa, T.; Okamoto, Y.; Kodama, Y.; Tanabe, T.; Shinkoda, Y.; Kawano, Y. Serum derivative of reactive oxygen metabolites (d-ROMs) in pediatric hemato-oncological patients with neutropenic fever. Pediatr. Blood Cancer 2010, 55, 91-94. [CrossRef]

16. Martarelli, D.; Pompei, P. Oxidative stress and antioxidant changes during a 24-hours mountain bike endurance exercise in master athletes. J. Sports Med. Phys. Fitness 2009, 49, 122-127.

17. Mochizuki, M.; Minowa, F.; Ishimoto, C.; Gin, A.; Ishioka, K.; Okubo, K. The effect of aging on biochemical markers in equine serum. J. Equine Vet. Sci. 2016, 42, 1-6. [CrossRef]

18. Gin, A.; Sato, T.; Tohei, A.; Miura, R.; Mizutani, H.; Amao, H.; Yamada, Y.; Kamiya, S.; Yosimura, I.; Mochizuki, M. Study of stress in dairy cattle during student, practical training on a farm. Jap. J. Vet. Res. 2018, 66, 63-70.

19. Brkljača Bottegaro, N.; Gotić, J.; Šuran, J.; Brozić, D.; Klobučar, K.; Bojanić, K.; Vrbanac, Z. Effect of prolonged submaximal exercise on serum oxidative stress biomarkers (d-ROMs, MDA, BAP) and oxidative stress index in endurance horses. BMC Vet. Res. 2018, 14, 216. [CrossRef] 
20. Tsubone, H.; Hanafusa, M.; Endo, M.; Manabe, N.; Hiraga, A.; Ohmura, H.; Aida, H. Effect of treadmill exercise and hydrogen-rich water intake on serum oxidative and anti-oxidative metabolites in serum of Thoroughbred horses. J. Equine Sci. 2013, 24, 1-8. [CrossRef]

21. Crowley, J.; Po, E.; Celi, P.; Muscatello, G. Systemic and respiratory oxidative stress in the pathogenesis and diagnosis of Rhodococcus equi pneumonia. Equine Vet. J. 2013, 45, 20-25. [CrossRef]

22. Sgorbini, M.F.; Bonelli, A.; Marmorini, R.P.; Biagi, G.; Corazza, M.; Pasquini, A. Maternal and neonatal evaluation of derivated reactive oxygen metabolites (d-ROMs) and biological antioxidant potential in the horse. Theriogenology 2015, 83, 48-51. [CrossRef] [PubMed]

23. Tsuzuki, N.; Sasaki, N.; Kusano, K.; Endo, Y.; Torisu, S. Oxidative stress markers in Thoroughbred horses after castration surgery under inhalation anesthesia. J. Equine Sci. 2016, 27, 77-79. [CrossRef]

24. Pasquini, A.; Luchetti, E.; Marchetti, V.; Cardini, G.; Iorio, E.L. Analytical performances of d-ROMs test and BAP test in canine plasma. Definition of the normal range in healthy Labrador dogs. Vet. Res. Commun. 2008, 32, 137-143. [CrossRef]

25. Fiore, F.; Spissu, N.; Sechi, S.; Cocco, R. Evaluation of oxidative stress in dairy cows with left displacement of abomasum. Animals 2019, 9, 966. [CrossRef]

26. Japan Racing Association. Equine Veterinary Medicine, 1st ed.; Equine Research Institute, Japan Racing Association; Midori Shobo Co., Ltd.: Tokyo, Japan, 2012; pp. 93-100. (In Japanease)

27. Tsuzuki, T.; Tsukioka, K.; Naito, H. Changes in the blood redox balance during a simulated duathlon race and its relationship with athletic performance. Physiol. Rep. 2019, 7, e14277. [CrossRef]

28. Kusano, K.; Yamazaki, M.; Kiuchi, M.; Kaneko, K.; Koyama, K. Reference range of blood biomarkers for oxidative stress in Thoroughbred racehorses (2-5 years old). J. Equine Sci. 2016, 27, 125-129. [CrossRef]

29. Takahashi, M.; Miyashita, M.; Park, J.H.; Kim, H.S.; Nakamura, Y.; Sakamoto, S.; Suzuki, K. The association between physical activity and sex-specific oxidative stress in older adults. J. Sport Sci. Med. 2013, 12, 571-578.

30. Pesce, M.; Tatangelo, R.; La Fratta, I.; Rizzuto, A.; Campagna, G.; Turli, C.; Ferrone, A.; Franceschelli, S.; Speranza, L.; Patruno, A.; et al. Aging-related oxidative stress: Positive effect of memory training. Neurosci. 2018, 370, 246-255. [CrossRef]

31. Alexander, S.; Irvine, C.H.G. Stress in the racing horse: Coping vs. not coping. J. Equine Sci. 1998, 9, 77-81. [CrossRef]

(C) 2020 by the authors. Licensee MDPI, Basel, Switzerland. This article is an open access article distributed under the terms and conditions of the Creative Commons Attribution (CC BY) license (http://creativecommons.org/licenses/by/4.0/). 